\title{
natureINSIGHT
}

\section{INTESTINAL NETWORKS IN HEALTH AND DISEASE}

16 June 2011 / Vol 474 / Issue No 7351

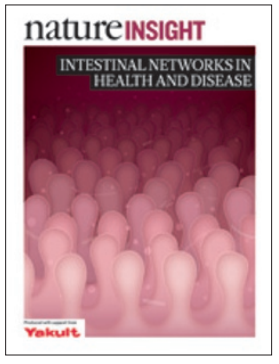

Cover illustration by Nik Spencer

Editor, Nature

Philip Campbell

Publishing

Nick Campbell

Insights Editor

Ursula Weiss

Production Editor

Nicola Bailey

Art Director

Kelly Buckheit Krause

Art Editor

Nik Spencer

Sponsorship

Gerard Preston

Production

Emilia Orviss

Marketing

Elena Woodstock,

Hannah Phipps

Editorial Assistant

Hazel Mayhew
( ur bowels have two major roles: the digestion and absorption of nutrients, and the maintenance of a barrier against the external environment. They fulfil these functions in the context of, and with help from, tens of trillions of resident microbes, known as the gut microbiota.

This Insight has as its topic the various relationships that contribute to keeping this complex system in balance, and that help to protect us from a wide spectrum of diseases, including chronic inflammatory bowel disease, colorectal cancer and metabolic disease. It explores the interactions between the environment and host genetics, between the type and amount of food we eat and the composition of the microbial community, and between the microbiota, the intestinal epithelium and the immune system. It also highlights the regulatory mechanisms that control the rapid and continuous renewal of epithelial cells in the intestinal lining from resident stem cells. It discusses the cellular and molecular pathways that help to maintain intestinal homeostasis, and explores the mechanisms that cause pathology and disease when these pathways fail.

We hope that these articles will contribute to a better understanding of the nature of these complex networks, and point to future strategies that will be successful in paving the way for more effective preventative and therapeutic measures, ultimately benefitting human health.

We are pleased to acknowledge the financial support of Yakult Honsha in producing this Insight. As always Nature carries sole responsibility for all editorial content and peer review.

Ulla Weiss

Insights Editor

\section{CONTENTS}

\section{REVIEWS}

298 Intestinal homeostasis and its breakdown in inflammatory bowel disease

Kevin J Maloy \& Fiona Powrie

307 Genetics and pathogenesis of inflammatory bowel disease Bernard Khor, Agnès Gardet \& Ramnik J Xavier

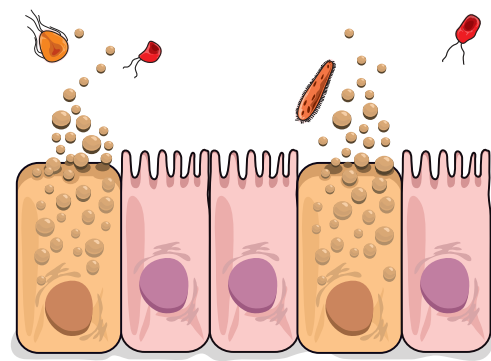

318 Microenvironmental regulation of stem cells in intestinal homeostasis and cancer

Jan Paul Medema \& Louis Vermeulen

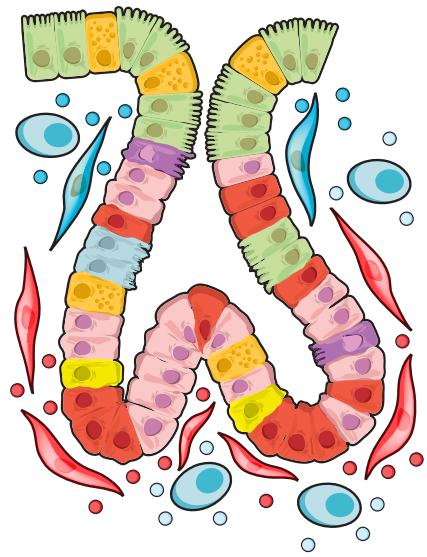

\section{PERSPECTIVE}

327 Human nutrition, the gut microbiome and the immune system Andrew L Kau, Philip P Ahern, Nicholas W Griffin, Andrew L Goodman \& Jeffrey / Gordon 